\title{
Synthesis of new hemiasterlin derivatives with $\alpha, \beta$-unsaturated carbonyl-thiophene groups in fragment $A$
}

\author{
Pham The Chinh ${ }^{1 *}$, Pham Thi Tham ${ }^{2}$, Nguyen Van Tuyen ${ }^{3}$ \\ ${ }^{1}$ Thai Nguyen University of Science \\ ${ }^{2}$ Hanoi University of Industry \\ ${ }^{3}$ Insitute of Chemistry, Vietnam Academy of Science and Technology \\ Received 19 February 2017; Accepted for publication 28 August 2017
}

\begin{abstract}
Hemiasterlin, an antimitotic tripeptide, exhibits cytotoxicity in the nanomolar range against a variety of cultured human and murine cell lines. For this reason, the synthesis of new hemiasterlin derivatives has attracted a lot of interest in the organic chemistry community recently. In this article, we synthesized new simplified derivatives of hemiasterlin in which the $\alpha, \alpha$-dimethylbenzylic group in fragment $\mathrm{A}$ is replaced by $\alpha, \beta$-unsaturated carbonyl-thiophene group. These new compounds will be prepared by classical peptide coupling approach between the carboxylic acid fragments A 12 and dipeptide 13. We expect that this derivative will possess interesting biological activities due to the high reactivity of the $\alpha, \beta$-unsaturated carbonyl group as Michael receptor with biological nucleophiles, such as DNA, RN5A, and enzymes.
\end{abstract}

Keywords. Synthesis, hemiasterlin, $\alpha, \beta$-unsaturated; tripeptides, nanomolar.

\section{INTRODUCTION}

Hemiasterlins belong to a family of naturally occurring tripeptides from marine sponges [1]. The important derivatives of hemiasterlin are hemiasterlin $\mathrm{A}$, hemiasterlin $\mathrm{B}$, and hemiasterlin $\mathrm{C}$, which were isolated from marine sponge Auletta and Cymbastella (figure 1) [2, 3]. These naturally occurring substances exhibited potent cytotoxicity in vitro against murine leukemia P388 and human breast, ovarian, colon, and lung cancer cell lines $[2,3,5]$. Hemiasterlins suppress microtubule depolymerization presumably by binding to the vinca alkaloid site of tubulin and causing mitotic arrest and cell death [4]. This active mechanism makes them very attractive molecules for new anticancer drugs. However, synthesis of the stereospecific amine group and especially the gemdimethyl moiety in segment A was proved to be highly problematic. To overcome this difficulty, several studies explored the modifications of segment A that eliminated the gem-dimethyl moiety [6]. Some derivatives from this approach $(\mathbf{7}, \mathbf{8})$ showed promising cytotoxic test results.
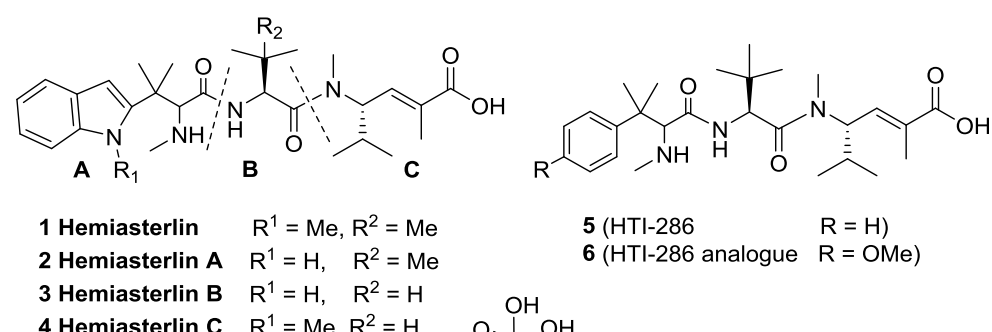

$\begin{array}{ll}5(\mathrm{HTI}-286 & \mathrm{R}=\mathrm{H}) \\ 6 & (\mathrm{HTl}-286\end{array}$

3 Hemiasterlin B $\quad \mathrm{R}^{1}=\mathrm{H}, \quad \mathrm{R}^{2}=\mathrm{H}$

4 Hemiasterlin C $R^{1}=M e, R^{2}=H$

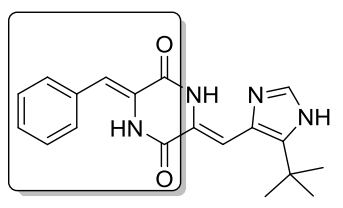

$7\left(\mathrm{IC}_{50}=15 \mathrm{nM}\right)$

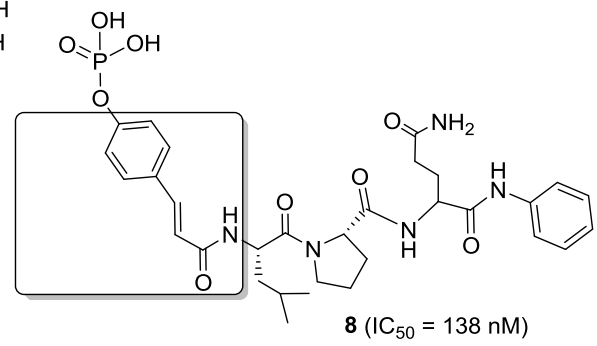

Figure 1: Examples of hemiasterlin derivatives and modification of segment A of hemiasterli 
Recently, Hayashi et al. and McMurray et al. prepared new synthetic peptides $(\mathbf{7}, \mathbf{8})$ with $\alpha, \beta$ unsaturated carbonyl systems which were thought to play a role in the remarkably strong cytotoxicity of these compounds [7, 8]. The $\alpha, \beta$-unsaturated carbonyl moieties are well-known Michael type acceptors. They are particularly reactive and interact strongly with electron-rich biological macromolecules such as DNA, protein, and enzyme, resulting in a wide range of biological effects including general toxicity, allergenic reactions, mutagenicity, and carcinogenicity. In this article, we report the synthesis of new derivatives of hemiasterlin with a novel direction in the construction of fragment $\mathrm{A}$ that contained $\alpha, \beta$ unsaturated-thiophene group.

\section{EXPERIMENTAL}

\subsection{General information}

All reactions were performed in the appropriate oven-dried glass apparatus and under nitrogen atmosphere. Unless otherwise stated, solvents and chemicals were obtained from commercial sources and used without further purification. Column chromatography was performed using silica gel $(60$ $\AA$, particle size 40-60 $\mu \mathrm{m})$. NMR spectra were recorded on a Bruker Avance $(500 \mathrm{MHz})$. Chemical shifts $(\delta)$ are given in parts per million (ppm) and coupling constants $(J)$ in hertz $(\mathrm{Hz})$. Mass spectrometry analysis (MS) were recorded on a Qexactive or a Q-TOF2 instrument. IR analysis was recorded on Perkin Elmer Spectrum Two.

\subsection{Synthesis of compound 11}

A solution of $N$-acetylglycine (10) $(500 \mathrm{mg}, 4.27$ mmol), thiophene-2-carbaldehyde (360 mg, 3.20 $\mathrm{mmol})$, acetic anhydride $(15 \mathrm{~mL})$, and fused sodium acetate $(350 \mathrm{mg}, 4.27 \mathrm{mmol})$ was heated at $90{ }^{\circ} \mathrm{C}$ with stirring for $12 \mathrm{~h}$. Afterwards, the acetic anhydride was evaporated in vacuum, the residue was extracted with $\mathrm{CH}_{2} \mathrm{Cl}_{2}$ and the combined $\mathrm{CH}_{2} \mathrm{Cl}_{2}$ extract was washed with brine, dried $\left(\mathrm{MgSO}_{4}\right)$. The solvent was removed in vacuum to give crude azalactone $\mathbf{1 1}$ which was purified by column chromatography on silica gel (nhexane/EtOAc 98/2) to afford pure azalactone 11 (328 mg, 53 \%). M.p. $168-170{ }^{\circ} \mathrm{C}$. IR (KBr): 3063 ; $1793 ; 1676 ; 1655 ; 1624 ; 1561 ; 1537 ; 1468 ; 1381$; $1305 ; 1247 ; 1189 ; 1017 ; 1003 ; 941 ; 896 ; 873 ; 824$; $766 \mathrm{~cm}^{-1} .{ }^{1} \mathrm{H}-\mathrm{NMR}\left(\mathrm{CDCl}_{3}, 500 \mathrm{MHz}\right) \delta \mathrm{ppm}: 8.41$ $(1 \mathrm{H}, \mathrm{s}, \mathrm{C} \underline{\mathrm{H}}=\mathrm{C}-)$; $7.66(1 \mathrm{H}$, brs, H-5'); $7.32(1 \mathrm{H}, \mathrm{brs}$, H-3'); 6.95 (1H, brs, H-4'); 2.45 (3H, s, $\left.\mathrm{CH}_{3}\right)$.

\subsection{Synthesis of compound 12}

A solution of azalactone $11(300 \mathrm{mg}, 1.55 \mathrm{mmol})$ in $\mathrm{NaOH} 1 \mathrm{~N}(5 \mathrm{~mL})$ was heated at $100{ }^{\circ} \mathrm{C}$ for $30 \mathrm{~min}$, followed by addition of a solution of $\mathrm{HCl} 12 \mathrm{~N}$ (5 $\mathrm{mL}$ ). After stirring for $4 \mathrm{~h}$, the solvent was evaporated in vacuum to give a residue which was extracted with EtOAc and the combined EtOAc extract was washed with brine and dried $\left(\mathrm{MgSO}_{4}\right)$. The solvent was removed in vacuum to give amide 12 which was purified by column chromatography on silica gel ( $n$-hexane/EtOAc: $95 / 5)$ to afford the pure desired product 12 (200 mg, $61 \%)$. M.p. 214$215{ }^{\circ} \mathrm{C}$. IR (KBr): 3422; 3288; 3121; 2915; 1687; $1640 ; 1624 ; 1558 ; 1527 ; 1471 ; 1519 ; 1302 ; 1244$; $1216 ; 1190 ; 1024 ; 941 ; 886 ; 869 ; 767 ; 749 ; 695$ $\mathrm{cm}^{-1} .{ }^{1} \mathrm{H}-\mathrm{NMR}\left(\mathrm{CDCl}_{3}, 500 \mathrm{MHz}\right) \delta$ ppm: $7.48(1 \mathrm{H}$, brs, H-4'); $7.15(1 \mathrm{H}$, brs, H-3'); $6.57(1 \mathrm{H}$, brs, H5); $6.44(1 \mathrm{H}, \mathrm{s}, \mathrm{H}-3) ; 2.11\left(3 \mathrm{H}, \mathrm{s}, \mathrm{CH}_{3}\right) .{ }^{13} \mathrm{C}-\mathrm{NMR}$ $\left(\mathrm{CDCl}_{3}, 125 \mathrm{MHz}\right) \delta \mathrm{ppm}: 170.6(\mathrm{CO}, \mathrm{Ac}), 166.4$ $(\mathrm{C}=\mathrm{O}), 149.1 \quad\left(\mathrm{C}-2^{\prime}\right), 144.5 \quad\left(\mathrm{C}-5^{\prime}\right), 121.7 \quad(\mathrm{NH}-$ $\underline{\mathrm{C}}=\mathrm{CH}) ; 120.6 \quad(\mathrm{NH}-\mathrm{C}=\underline{\mathrm{CH}}), 115.5 \quad\left(\underline{\mathrm{C}}-3^{\prime}\right), 111.9$ (C-4'), $22.0\left(\underline{\mathrm{CH}}_{3}\right)$.

\subsection{Synthesis of compounds $14 a, b$}

To a solution of compound 13a (100 mg, 0.24 $\mathrm{mmol}$ ), EDC (50 mg, $0.26 \mathrm{mmol})$, HOBt $(35 \mathrm{mg}$, $0.26 \mathrm{mmol})$ and $i$-PrNHEt (124 $\mathrm{mg}, 0.48 \mathrm{mmol})$ in DMF (3 mL) was added compound 12 (55 mg, 0.26 $\mathrm{mmol}$ ) and the solution was stirred at room temperature for $12 \mathrm{~h}$. The reaction mixture was partitioned between water and EtOAc and extracted with EtOAc. The combined EtOAc extract was washed with brine, dried over $\mathrm{MgSO}_{4}$, filtered, and concentrated under reduced pressure to give the crude product. This material was purified by column chromatography on silica gel (hexane/EtOAc: 80/20) to obtain pure compound 14a (59 $\mathrm{mg}, 50 \%)$. The compound 14b (62\%) was prepared in the same way using acid $\mathbf{1 2}$ as starting material.

Compound 14a. White solid in $50 \%$ yield, m.p. 96-98 ${ }^{\circ} \mathrm{C}$. IR (KBr): 3292; 2962; 2878; 1700; 1650; $1529 ; 1370 ; 1270 ; 1240 ; 1109 ; 752 \mathrm{~cm}^{-1} .{ }^{1} \mathrm{H}-\mathrm{NMR}$ $\left(\mathrm{CDCl}_{3}, 500 \mathrm{MHz}\right) \delta$ ppm: $7.52(1 \mathrm{H}, \mathrm{s}, \mathrm{H}-5$ '); 6.73 (1H, brs, H-3'); 6.62 (1H, brs, H-4'); $6.57(1 \mathrm{H}, \mathrm{s}, \mathrm{H}-$ 15); $6.48(1 \mathrm{H}, \mathrm{s}, \mathrm{H}-3) ; 4.54(1 \mathrm{H}, \mathrm{s}, \mathrm{H}-10) ; 4.39(1 \mathrm{H}$, d, $J=9.0 \mathrm{~Hz}, \mathrm{H}-4) ; 4.13-4.20\left(2 \mathrm{H}, \mathrm{m}, \mathrm{CH}_{2} \mathrm{Et}\right) ; 2.10$ $\left(3 \mathrm{H}, \mathrm{s}, \mathrm{CH}_{3} \mathrm{Ac}\right) ; 1.92\left(3 \mathrm{H}, \mathrm{s}, \mathrm{CH}_{3}-\mathrm{C} 2\right) ; 1.77-1.82$ $(1 \mathrm{H}, \mathrm{m}, \mathrm{H}-5) ; 1.27\left(3 \mathrm{H}, \mathrm{t}, J=7.0 \mathrm{~Hz}, \mathrm{CH}_{3} \mathrm{Et}\right) ; 0.97$ $(9 \mathrm{H}, \mathrm{s}, \mathrm{H}-12) ; 0.86(6 \mathrm{H}, \mathrm{d}, J=7.5 \mathrm{~Hz} ; \mathrm{H}-6, \mathrm{H}-7)$. ${ }^{13} \mathrm{C}-\mathrm{NMR}\left(\mathrm{CDCl}_{3}, 125 \mathrm{MHz}\right) \delta \mathrm{ppm}: 169.8(\mathrm{C}-9)$; $168.2(\mathrm{C}=\mathrm{O}$ Ac); 164.6 (COOEt); 162.4 (C-13); 149.7 (C-2'); 143.9 (C-5'); 139.6 (C-14); 129.8 (C3); 127.1 (C-2); 114.3 (C-15); 113.7 (C-3'); 112.0 
(C-4'); 61.1 (C-10); 60.7 (C-4); $52.7\left(\mathrm{CH}_{2} \mathrm{Et}\right) ; 36.4$ (C-11); 34.9 (C-5); 32.7 ( CH$\left._{3} \mathrm{Ac}\right) ; 26.6$ (C-12); 18.6 (C-6, C-7); 14.1 ( $\left.\underline{\mathrm{CH}}_{3} \mathrm{Et}\right) ; 13.1\left(\underline{\mathrm{CH}}_{3}-\mathrm{C}-2\right)$. HRMS (ESI) $[\mathrm{M}+\mathrm{Na}]^{+}$: calc. for $\mathrm{C}_{25} \mathrm{H}_{37} \mathrm{~N}_{3} \mathrm{NaO}_{5} \mathrm{~S}$ : 514.2346; Found: 514.2349.

Compound 14b. White solid in $62 \%$ yield, m.p. 93-94 ${ }^{\circ} \mathrm{C}$. IR (KBr): 3278; 2960; 2932; 2871; 1710; $1691 ; 1665 ; 1620 ; 1510 ; 1475 ; 1410 ; 1369 ; 1281$; $1248 ; 1100 ; 1026 ; 981 ; 753 \mathrm{~cm}^{-1} .{ }^{1} \mathrm{H}-\mathrm{NMR}\left(\mathrm{CDCl}_{3}\right.$, $500 \mathrm{MHz}) \delta$ ppm: $7.51(1 \mathrm{H}$, brs, H-5'); $6.89(1 \mathrm{H}$, brs, H-3'); $6.78(1 \mathrm{H}$, brs, H-4'); $6.62(1 \mathrm{H}, \mathrm{dd}, J=$ 1.5, 9.0 Hz,H-3); $6.46(1 \mathrm{H}, \mathrm{s}, \mathrm{H}-15) ; 5.01(1 \mathrm{H}, \mathrm{s}, \mathrm{H}-$ $10) ; 4.92(1 \mathrm{H}, \mathrm{d}, J=9.5 \mathrm{~Hz}, \mathrm{H}-4) ; 4.16-4.22(2 \mathrm{H}, \mathrm{m}$, $\left.\mathrm{C}_{2} \mathrm{Et}\right) ; 3.0$ (3H, s, H-8); 2.19 (3H, s, $\left.\mathrm{CH}_{3} \mathrm{Ac}\right) ; 1.91$ $\left(3 \mathrm{H}, \mathrm{s}, \mathrm{CH}_{3}-\mathrm{C}-2\right) ; 1.88-1.89(1 \mathrm{H}, \mathrm{m}, \mathrm{H}-5) ; 1.27(3 \mathrm{H}$, $\left.\mathrm{t}, J=7.0 \mathrm{~Hz}, \mathrm{C}_{3} \mathrm{Et}\right) ; 0.99$ (9H, s, H-12); $0.84(6 \mathrm{H}$, $\mathrm{d}, J=7.5 ; \mathrm{Hz}, \mathrm{H}-6, \mathrm{H}-7) .{ }^{13} \mathrm{C}-\mathrm{NMR}\left(\mathrm{CDCl}_{3}, 125\right.$ MHz) $\delta$ ppm: 170.2 (C-9); 167.7 ( $\underline{C}=\mathrm{O}$ Ac); 164.1 (COOEt); 162.0 (C-13); 149.8 (C-2'); 143.7 (C-5'); 138.2 (C-14); 132.6 (C-3); 127.0 (C-2); 121.2 (C15); 113.8 (C-3'); 112.4 (C-4'); 60.9 (C-10); 56.2 (C-4); $55.7\left(\mathrm{CH}_{2} \mathrm{Et}\right) ; 35.2$ (C-11); 34.5 (C-5); 31.1 (C-8); $29.9\left(\underline{\mathrm{C}}_{3} \mathrm{Ac}\right) ; 26.4$ (C-12); 18.7 (C-6, C-7); $14.3 \quad\left(\underline{\mathrm{CH}}_{3} \quad \mathrm{Et}\right) ; 13.8 \quad\left(\underline{\mathrm{CH}}_{3} \mathrm{C}-2\right) . \quad$ HRMS (ESI) $[\mathrm{M}+\mathrm{Na}]^{+}:$calc. for $\mathrm{C}_{26} \mathrm{H}_{39} \mathrm{~N}_{3} \mathrm{NaO}_{5} \mathrm{~S}$ : 528.2503; Found: 528.2511.

\subsection{Synthesis of compound $15 a, b$}

To a cooled $\left(0{ }^{\circ} \mathrm{C}\right)$ solution of $14 \mathbf{a}(50 \mathrm{mg}, 0.102$ $\mathrm{mmol})$ in $2 \mathrm{~mL} \mathrm{MeOH} / \mathrm{H}_{2} \mathrm{O}(2: 1)$ was added $\mathrm{LiOH}$ (25 mg, $1.02 \mathrm{mmol}$ ). The cooling bath was removed, and the resulting mixture was stirred at room temperature for $10 \mathrm{~h}$. The solvent was removed in vacuum and the residue was dissolved in a small amount of $\mathrm{H}_{2} \mathrm{O}$. The aqueous solution was cooled to $0{ }^{\circ} \mathrm{C}$, and acidified to $\mathrm{pH}$ 5.5-6.0 with aqueous hydrochloride acid solution $(\mathrm{pH}=2)$. Afterward, the reaction mixture was extracted with EtOAc. The combined EtOAc extract was washed with brine, dried over $\mathrm{MgSO}_{4}$, filtered, and concentrated under reduced pressure to give pure compound 15a (45 mg, $95 \%)$. The compound $\mathbf{1 5 b}(97 \%)$ was prepared in the same way from $\mathbf{1 4 b}$.

Compound 15a. White solid in $95 \%$ yield, m.p. $120-122{ }^{\circ} \mathrm{C}$. IR (KBr): 3410; 3279; 2963; 2874; $1649 ; 1519 ; 1385 ; 1370 ; 1268 ; 1242 ; 1150 ; 1021$; $751 \mathrm{~cm}^{-1} .{ }^{1} \mathrm{H}-\mathrm{NMR}\left(\mathrm{CD}_{3} \mathrm{OD}, 500 \mathrm{MHz}\right) \delta \mathrm{ppm}: 7.69$ $(1 \mathrm{H}, \mathrm{d}, J=1.5 \mathrm{~Hz}, \mathrm{H}-5$ '); $7.12(1 \mathrm{H}, \mathrm{s}, \mathrm{H}-15) ; 6.73$ $\left(1 \mathrm{H}, \mathrm{d}, J=3.5 \mathrm{~Hz}, \mathrm{H}^{-}{ }^{\prime}\right)$; $6.60(1 \mathrm{H}, \mathrm{dd}, J=1.5,10.0$ $\mathrm{Hz}, \mathrm{H}-3) ; 6.58(1 \mathrm{H}, \mathrm{dd}, J=1.5,3.5 \mathrm{~Hz}, \mathrm{H}-4$ '); 4.50 $(1 \mathrm{H}, \mathrm{s}, \mathrm{H}-10) ; 4.39(1 \mathrm{H}, \mathrm{dd}, J=1.5,10.0 \mathrm{~Hz}, \mathrm{H}-4)$; $2.22\left(3 \mathrm{H}, \mathrm{s}, \mathrm{C}_{3} \mathrm{Ac}\right) ; 1.92\left(3 \mathrm{H}, \mathrm{s}, \mathrm{C}_{3}-\mathrm{C}-2\right) ; 1.81-$ 1.84 (1H, m, H-5); 1.00 (9H, s, H-12); 0.96 (3H, d, $J$ $\left.=6.5 \mathrm{~Hz}, \underline{\mathrm{C}}_{3} \mathrm{H}-6\right) ; 0.91(3 \mathrm{H}, \mathrm{d}, J=6.5 \mathrm{~Hz}, \mathrm{H}-7)$.
${ }^{13} \mathrm{C}-\mathrm{NMR}\left(\mathrm{CD}_{3} \mathrm{OD}, 125 \mathrm{MHz}\right) \delta \mathrm{ppm}: 173.4(\mathrm{C}-1)$; 171.8 (C-9); 166.5 (ㅁ Ac); 149.1 (C-2'); 146.7 (C5'); 140.2 (C-3); 131.4 (C-14); 126.7 (C-3); 119.5 (C-15); 116.2 (C-4'); 113.2 (C-3'); 62.2 (C-10); 54.5 (C-4); 36.2 (C-11); 33.6 (C-5); 27.1 (C-12); 22.7 $\left(\mathrm{CH}_{3} \mathrm{Ac}\right) ; 19.3$ (C-6); 19.1 (C-7); $13.3\left(\mathrm{CH}_{3}-\mathrm{C}-2\right)$. HRMS (ESI) $[\mathrm{M}+\mathrm{Na}]^{+}$: calc. for $\mathrm{C}_{23} \mathrm{H}_{33} \mathrm{~N}_{3} \mathrm{NaO}_{5} \mathrm{~S}$ : 486.2033; Found: 486.2042.

Compound 15b. White solid in $97 \%$ yield, m.p. 128-129 ${ }^{\circ} \mathrm{C}$. IR (KBr): 3420; 3280; 2961; 2930; $2875 ; 1697 ; 1620 ; 1514 ; 1478 ; 1410 ; 1369 ; 1270$; $1244 ; 1077 ; 1022 ; 980 ; 754 \mathrm{~cm}^{-1} .{ }^{1} \mathrm{H}-\mathrm{NMR}\left(\mathrm{CD}_{3} \mathrm{OD}\right.$, $500 \mathrm{MHz}) \delta$ ppm: $7.60(1 \mathrm{H}, \mathrm{d}, J=1.5 \mathrm{~Hz}, \mathrm{H}-5$ '); $7.12(1 \mathrm{H}, \mathrm{s}, \mathrm{H}-15) ; 6.76(2 \mathrm{H}, \mathrm{dd}, J=1.0,3.5 \mathrm{~Hz}, \mathrm{H}-$ 3', H-4'); $6.58(1 \mathrm{H}, \mathrm{dd}, J=1.5,9.0 \mathrm{~Hz}, \mathrm{H}-3) ; 5.08$ $(1 \mathrm{H}, \mathrm{s}, \mathrm{H}-10) ; 4.97(1 \mathrm{H}, \mathrm{d}, J=9.5 \mathrm{~Hz}, \mathrm{H}-4) ; 3.06$ $(3 \mathrm{H}, \mathrm{s}, \mathrm{H}-8) ; 2.21\left(3 \mathrm{H}, \mathrm{s}, \mathrm{CH}_{3} \mathrm{Ac}\right) ; 1.99-2.03(1 \mathrm{H}$, m, H-5); $1.91\left(3 \mathrm{H}, \mathrm{s}, \underline{\mathrm{C}}_{3}-\mathrm{C}-2\right) ; 1.02\left(9 \mathrm{H}, \mathrm{s}, 3 \mathrm{C}_{3}\right.$ $\mathrm{H}-12) ; 0.91(3 \mathrm{H}, \mathrm{d}, J=6.5 \mathrm{~Hz}, \mathrm{H}-6) ; 0.86(3 \mathrm{H}, \mathrm{d}, J$ $=6.5 \mathrm{~Hz}, \mathrm{H}-7) \cdot{ }^{13} \mathrm{C}-\mathrm{NMR}\left(\mathrm{CDCl}_{3}, 125 \mathrm{MHz}\right) \delta \mathrm{ppm}$ : 173.5 (C-1); 173.3 (C-9); $173.1(\mathrm{C}=\mathrm{O}$ Ac); 166.7

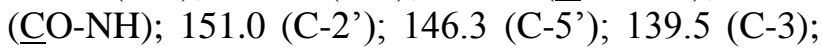
133.8 (C-14); 126.6 (C-2); 119.6 (C-15); 116.2 (C4'); 113.1 (C-3'); 58.9 (C-10); 56.8 (C-4); 37.0 (C11); 31.7 (C-8); 30.9 (C-5); 26.8 (C-12); $22.7\left(\mathrm{CH}_{3}\right.$ Ac); 19.7 (C-6); 19.2 (C-7); $14.0\left(\right.$ CH$\left._{3}-\mathrm{C}-2\right)$.

\section{RESULTS AND DISCUSSION}

Recently, we synthesized new hemiasterlin analogues in which the $\alpha, \alpha$-dimethylbenzylic group and amino NHMe moiety were replaced respectively by a $\alpha, \beta$ unsaturated aryl and an amide NHAc group leading to the suppression of one chiral center [10]. However, derivatives of hemiasterlin containing 2-thiophenyl group moiety have not been investigated. As a part of our ongoing work, we continue to focus on the new hemiasterlin analogues. For this purpose, we investigated the synthesis of new hemiasterlin derivatives in which the $\alpha, \alpha$-dimethylbenzylic group in fragment $\mathrm{A}$ is replaced by $\alpha, \beta$-unsaturated thiophen-2-yl group. These new compounds will be prepared by classical peptide coupling approach between the carboxylic acid fragments A $\mathbf{1 2}$ and dipeptide 13 [10]. A general procedure for the synthesis of compound $\mathbf{1 2}$ is outlined in Scheme 1 [10]. Compound $\mathbf{1 2}$ was synthesized from $N$-acetyl glycine (10) through two steps. The first step was condensation of $\mathrm{N}$-acetyl glycine (10) with furan-2carbaldehyde using sodium acetate in the presence of acetic anhydride at $90{ }^{\circ} \mathrm{C}$ for $12 \mathrm{~h}$ affording azalactone $\mathbf{1 1}$ in $80 \%$ yield $[9,10]$. Finally, azlactone 11 was hydrolyzed in aqueous sodium hydroxide, followed by treatment with hydrochloric acid (12 N) at $100{ }^{\circ} \mathrm{C}$ for $4 \mathrm{~h}$ to give compound $\mathbf{1 2}$ in $61 \%$ yield. 


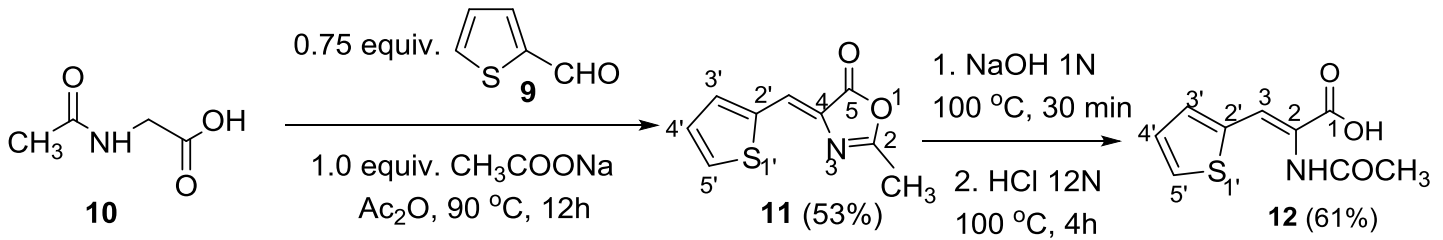

\section{Scheme 1}

The hemiasterlins 15a,b were prepared in two steps after peptide coupling reactions of $\mathbf{1 3} \mathbf{a}, \mathbf{b}$ with amides $\mathbf{1 2}$ followed by the saponification of ester using a $1 \mathrm{~N}$ lithium hydroxide solution (scheme 2). The HRMS (ESI) of compound 14a showed a pseudo-molecular ion peak at $\mathrm{m} / \mathrm{z}$ 514.2349, corresponding to the molecular formula of $\mathrm{C}_{25} \mathrm{H}_{37} \mathrm{~N}_{3} \mathrm{NaO}_{5} \mathrm{~S}$. The ${ }^{1} \mathrm{H}-\mathrm{NMR}$ showed signals of a thiophene ring at $7.51\left(1 \mathrm{H}\right.$, brs, $\left.\mathrm{H}-5^{\prime}\right) ; 6.89(1 \mathrm{H}$, brs, H-3') and 6.78 (1H, brs, H-4'). The ${ }^{13} \mathrm{C}-\mathrm{NMR}$ revealed its function groups, as follows: an thiophene heterocycles $149.7,113.7$ and 112.0; four carbonyls $169.8,168.2,164.6$ and 162.4; two double bonds 139.6, 114.3, 129.8 and 126.6; seven metyls including one metyl of acetyl, one vinylmetyl, a gem-dimethyl group, one isopropyl and one tert-butyl group. All spectral data thus confirmed the structure of hemiasterlin 14a. The structure of hemiasterlins 14b and 15a,b were elucidated the same way by IR, NMR and MS spectroscopic methods.

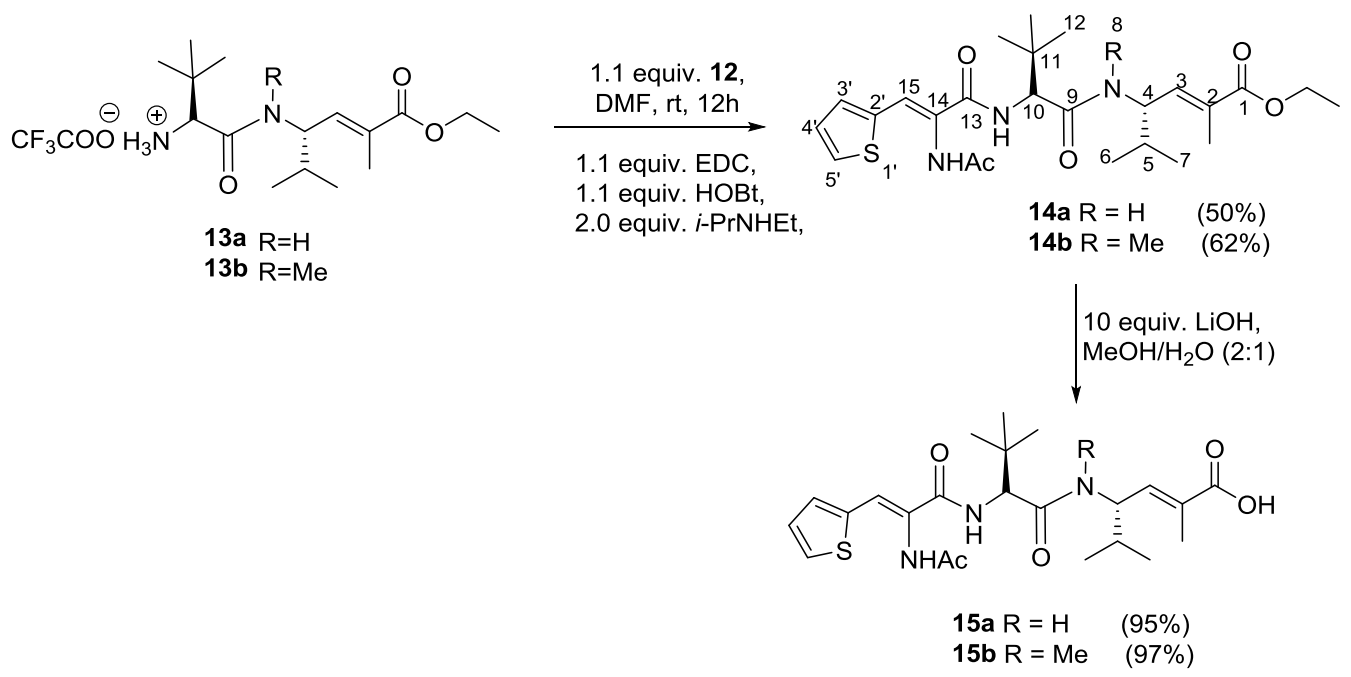

Scheme 2

In conclusion, a successful synthesis of new modified hemiasterlin derivatives was achieved in which the $\alpha, \alpha$-dimethylbenzylic group and amino $\mathrm{NHMe}$ moiety were replaced by $\alpha, \beta$-unsaturated carbonyl-thiophene and amide $\mathrm{NH}-\mathrm{Ac}$ group, respectively.

\section{REFERENCES}

1. Talpir R., Benayahu Y., Kashman Y., Pannell L., Schleyer M. Hemiasterlin and geodiamolide TA- two new cytotoxic peptides from the marine sponge Hemiasterella minor, Tetrahedron Lett., 35, 44534456 (1994).

2. Gamble W. R., Durso N. A., Fuller R. W., Westergaard C. K., Johnson T. R., Sackett, D. L.; Hamel E., Cardellina II J. H., Boyd M. R. Cytotoxic and tubulin-interactive hemiasterlins from Auletta sp. and Siphonochalina spp. Sponges, Bioorg. Med. Chem., 7, 1611-1615 (1999).

3. Coleman J. E., Silva E. D., Kong F., Andersen R. J., Allen T. M. Cytotoxic peptides from the marine sponge Cymbastela sp., Tetrahedron, 51, 10653 (1995).

4. Anderson H. J., Coleman J. E., Andersen R. J., Roberge M. Cytotoxic peptides hemiasterlin, hemiasterlin $A$ and hemiasterlin $B$ induce mitotic arrest and abnormal spindle formation, Cancer Chemother. Pharmacol., 39, 223-226 (1997).

5. Yamashita A., Norton E. B., Kaplan J. A., Niu C., Loganzo F., Hernand R., Beyer C. F., Annable T., Musto S., Discafani C., Zask A., Ayral-Kaloustian A. Synthesis and activity of novel analoges of hemiasterlin as inhibitors of tubulin polymerization: 
VJC, 55(4), 2017

modification of the A segment, Bioorg. Med. Chem. Lett., 14, 5317-5322 (2004).

6. Niew J. A., Coleman J. E., Wallace D. J., Piers E., Lim L. Y., Roberge M., Andersen R. J. Synthesis and Antimitotic/Cytotoxic Activity of Hemiasterlin Analogues, J. Nat. Prod., 6, 183 (2003).

7. Hayashi Y. et al. Synthesis and Structure-Activity Relationship Study of Antimicrotubule Agents Phenylahistin Derivatives with a Didehydropiperazine-2,5-dione Structure, J. Med. Chem., 55, 1056-1071 (2012).

8. Pijus K. M., Zhiyong R., Xiaomin C., Chiyi X., McMurray J. S. Structure-Affinity Relationships of Glutamine Mimics Incorporated into
Synthesis of new hemiasterlin derivatives with...

Phosphopeptides Targeted to the $\mathrm{SH}_{2}$ Domain of Signal Transducer and Activator of Transcription 3, J. Med. Chem., 52, 6126-6141 (2009).

9. Thomas C., Jodie B., Flavio C. One-pot process to Z$\alpha$-benzoylamino-acrylic acid methyl esters via potassium phosphate-catalyzed Erlenmeyer reaction, Tetrahedron Lett., 51, 625-628 (2010).

10. Chinh Pham The, Tuyet Anh Dang Thi, Thi Phuong Hoang, Quoc Anh Ngo, Duy Tien Doan, Thu Ha Nguyen Thi, Tham Pham Thi, Thu Ha Vu Thi, M. Jean, P. van de Weghe, Tuyen Nguyen Van. Synthesis of New simplified hemiasterlin derivatives with $\alpha, \beta$-unsaturated carbonyl moiety, Bioorganic \& Medicinal Chemistry Letters, 24, 2244-2246 (2014).

\section{Corresponding author: Pham The Chinh}

Thai Nguyen University of Science, Thai Nguyen University

Luong Ngoc Quyen Str. Tan Thinh, Thai Nguyen

E-mail: chinhpt@tnus.edu.vn, Telephone: 0988113933. 